UDC 519.654+539.31 Вестник СПбГУ. Прикладная математика. Информатика... 2020. Т. 16. Вып. 4 MSC 74S60

\title{
Statistical criteria for the limits of application of Hooke's law
}

\section{A. V. Orekhov}

St. Petersburg State University, 7-9, Universitetskaya nab., St. Petersburg, 199034, Russian Federation

For citation: Orekhov A. V. Statistical criteria for the limits of application of Hooke's law. Vestnik of Saint Petersburg University. Applied Mathematics. Computer Science. Control Processes, 2020, vol. 16, iss. 4, pp. 391-401. https://doi.org/10.21638/11701/spbu10.2020.404

Modern methods for studying the stress-strain state of solids use graphical methods based on a stress-strain curve to determine the transition from elastic deformation to plastic deformation. However, this approach is not formal and it is intended only for when stress is a function of strain in the one-dimensional case. Cases, when strain is a function of the stress, are also of practical importance. The purpose of the study is to develop formal rules for determining the limits of applicability of Hooke's law. The proposed analytical methods for determining the transition from elastic deformation to plastic deformation are based on consistent statistical sequential. In this article, quadratic forms are derived for calculating the point at which the type of an increasing monotonous numerical sequence changes from linear to non-linear type. With the help of these quadratic forms, statistical criteria (approximation-estimation tests) are constructed to determine the limits of applicability for Hooke's law. These boundaries are defined as Markov moments. The novelty of the results shows that it is possible to determine the yield point without visualizing the experimental data. The numerical example of the application of a parabolic approximationestimation test is provided. From the results of this experiment, it can be concluded that the analytical determination of the limits of applicability of Hooke's law coincides with a visual assessment. Approximation-estimation tests provide an opportunity to determine the limits of applicability of Hooke's law analytically.

Keywords: Hooke's law, stress, strain, approximation-estimation test, least squares method, Markov moments.

1. Introduction. The primary purpose of studying the properties of materials when exposed to external forces is to establish a relationship between stresses and strains. Sometimes displacements and deformations are determined by stresses and forces, and sometimes vice versa, forces, and stresses are determined by displacements and deformations. The purest form of such a dependence arises during elastic deformations and is expressed by Hooke's law, which states that stress is proportional to deformation $[1,2]$.

For example, for uniaxial tension (compression) along the $z$-axis, this law can be expressed by the simple formula $\sigma_{z}=E \varepsilon_{z}$, where $\sigma_{z}$ is the longitudinal stress, $\varepsilon_{z}$ is the relative elongation, $E$ is proportionality coefficient. This coefficient is called Young's modulus (for a fixed isotropic and homogeneous material, the coefficient $E$ is a constant value). It is important to note that during axial compression of the cylinder, in addition to longitudinal shortening, transverse elongation occurs. However, in the transverse direction, there is no stress. Therefore, the one-dimensional form of Hooke's law generally cases insufficient. Experimental studies have shown that under uniaxial stress in solids, triaxial deformation occurs (except for individual cases of anisotropy) [3].

Transverse deformation under elastic tension (compression) is characterized by the

(C) Санкт-Петербургский государственный университет, 2020 
Poisson coefficient of $\nu$, which is equal to the ratio of transverse and longitudinal deformation with the opposite sign. Another important quantity is the shear modulus $G$, which characterizes the material's ability to resist shear. The following relationship determines the shear modulus:

$$
G=\frac{\tau}{\gamma}
$$

where $\tau$ is the shear stress, $\gamma$ is shear deformation. In a homogeneous isotropic material, the shear modulus is connected with the Young's modulus through the Poisson's ratio:

$$
G=\frac{E}{2(1+\nu)},
$$

here $\nu$ is the value of the Poisson's ratio for a given material $[1,2]$.

The shear modulus, Young's modulus, and Poisson's ratio are quantities characterizing the elastic properties of a material. All of them are used in the generalized Hooke's law [2]. For an isotropic material, this law can be represented:

$$
\begin{array}{rlrl}
\varepsilon_{x} & =\frac{1}{E}\left[\sigma_{x}-\nu\left(\sigma_{y}+\sigma_{z}\right)\right], & \gamma_{x y} & =\frac{\tau_{x y}}{G}, \\
\varepsilon_{y}=\frac{1}{E}\left[\sigma_{y}-\nu\left(\sigma_{z}+\sigma_{x}\right)\right], & \gamma_{y z}=\frac{\tau_{y z}}{G}, \\
\varepsilon_{z}=\frac{1}{E}\left[\sigma_{z}-\nu\left(\sigma_{x}+\sigma_{y}\right)\right], & \gamma_{x z}=\frac{\tau_{x z}}{G} .
\end{array}
$$

Continuous medium and internal stresses are abstract scientific concepts. They differ from real crystalline lattices and laws of interatomic interaction. Therefore, Hooke's law is a model approximation of physical processes in solids. However, experimentally shown that this law is observed with sufficient accuracy for the vast majority of materials, but only within specified limits. The limits of applicability of Hooke's law are limited to the onset of significant deviations from the linear relationship between stresses and strains. That is, if the relationship between deformation and stress is linear, then the calculation of strength, stiffness, and stability is based on the paradigm of Hooke's law. If the relation between stresses and strains ceases to be linear, then Hooke's law becomes inapplicable [3]. Such situations arise both in experiments and in theoretical studies.

If we study the dependence of the stress on the strain, the transition from an elastic to a plastic state is characterized by a change in the type of increase in stress from linear to logarithmic, for example [4,5]. If the strain is a function of stress, then the transition from an elastic to a plastic state is characterized by a change in the type of strain growth from linear to parabolic or exponential, for example [6, 7].

The test results for the strength of various materials or the numerical modeling of their properties are usually presented in the form of tabular functions. In most cases, their analytical form is unknown. Currently used graphical methods for determining the elastic-plastic zone boundaries using the stress-strain curve are relatively primitive. They are intended only when the stress is a function of strain [8, 9]. Cases when strain is a function of the stress are also it is crucial.

In this regard, it is of practical interest to obtain statistical criteria that make it possible to determine the moment when the nature of the monotonous increase in the tabulated value goes from linear to non-linear type.

2. Four classes of approximating functions. We will build these criteria in the form of statistics based on a comparison of the quadratic errors of approximation of a 
numerical sequence in four classes of real functions: linear $-f(x)=a x+b$, incomplete parabolic (without linear term) $-f(x)=c x^{2}+d$, logarithmic $-f(x)=g \ln (x+1)+h$ and exponential $-f(x)=p e^{x}+q$. We consider mappings defined on a discrete subset of points $\left\{t_{0}, t_{1}, \ldots, t_{n}, \ldots\right\}$ of the number line $\mathbb{R}$. If all these points are equidistant, i. e. for $\forall n: t_{n}-t_{n-1}=T$, then such functions are called functions of discrete argument and are denoted as $f[n T]$, where $T$ is the period of discreteness. The transition from linear to non-linear dependence does not depend on the scale. Therefore, due to the similarity transformation, the discreteness period $T$ can be to the unit one and consider in the further the lattice functions as numeric sequences $y_{n}=\left\{y_{0}, y_{1}, \ldots, y_{n}, \ldots\right\}$.

We will accept the agreement that the sequence $y_{n}$ is non-negative, monotonically increasing, and a priori $y_{n}$ first changes "linearly" and then "nonlinearly". For further constructions, it is necessary to agree on the precise understanding of the terms: "linear increase" and "nonlinear increase" of a numerical sequence. In this case, the evaluation of the nature of the change in $y_{n}$ is implied by the local. Not overall values $y_{n}$ but only by several elements $y_{0}, y_{1}, \ldots, y_{k-1}$.

We use the concept of an approximating function [10, 11]. The approximation nodes for the $y_{n}$ numerical sequence are ordered pairs $\left(i, y_{i}\right)$, where $i$ is a natural argument, $y_{i}$ is the corresponding sequence values of $y_{n}$. Since the subscript of the sequence $y_{n}$ uniquely identifies natural argument, then the approximation node $\left(i, y_{i}\right)$ will be identified with the element of the sequence $y_{i}$, we will call them "natural nodes". The mapping $f(x)$ from the class of function $X$ is an approximating function for the natural nodes $y_{0}, y_{1}, \ldots, y_{k-1}$, if it is the most is close to these points (in a certain sense) among all the mappings from $X$. The segment of the real axis $\left[y_{0}, y_{k-1}\right]$ is called "a current interval of approximation". It is clear that all natural nodes $y_{0}, y_{1}, \ldots, y_{k-1}$, belong to $\left[y_{0}, y_{k-1}\right]$.

The sum of the squares of the differences $y_{n}$ and $f(x)$ with the corresponding the values of the natural argument is called the quadratic error of the approximation of the number sequence $y_{n}$ by the function $f(x)$ at the natural nodes $y_{0}, y_{1}, \ldots, y_{k-1}$ :

$$
\delta^{2}=\sum_{i=0}^{k-1}\left(f(i)-y_{i}\right)^{2} .
$$

A real function $f(x)$ of a certain class $X$ approximates the numerical sequence $y_{n}$ using the method of least squares, if the following for quadratic form $\delta_{f}^{2}$ is true:

$$
\delta_{f}^{2}=\min _{f \in X} \sum_{i=0}^{k-1}\left(f(i)-y_{i}\right)^{2} .
$$

Such a minimum can always be found as $\delta_{f}^{2}$ is a positive definite quadratic form $[12,13]$.

The quadratic errors for the linear, incomplete parabolic, logarithmic and exponential approximations for the natural nodes $y_{0}, y_{1}, \ldots, y_{k-1}$ are respectively equal to:

$$
\begin{gathered}
\delta_{l}^{2}(k)=\sum_{i=0}^{k-1}\left(a \cdot i+b-y_{i}\right)^{2}, \quad \delta_{q}^{2}(k)=\sum_{i=0}^{k-1}\left(c \cdot i^{2}+d-y_{i}\right)^{2}, \\
\delta_{n}^{2}(k)=\sum_{i=0}^{k-1}\left(g \cdot \ln (i+1)+h-y_{i}\right)^{2}, \quad \delta_{e}^{2}(k)=\sum_{i=0}^{k-1}\left(p \cdot e^{i}+q-y_{i}\right)^{2} .
\end{gathered}
$$

Let $m=\min \left(\delta_{l}^{2}(k), \delta_{q}^{2}(k), \delta_{n}^{2}(k), \delta_{e}^{2}(k)\right)$. 
We will assume by definition that increasing the number sequence $y_{n}$ over the natural nodes $y_{0}, y_{1}, \ldots, y_{k-1}$ is linear if $m=\delta_{l}^{2}(k)$. Respectively: an increase in $y_{n}$ is parabolic in nature, if $m=\delta_{q}^{2}(k)$, an increase in $y_{n}$ is logarithmic, if $m=\delta_{n}^{2}(k)$, an increase in $y_{n}$ is exponential, if $m=\delta_{e}^{2}(k)$.

3. Construction of "approximation-estimating tests". When constructing quadratic forms of "approximation-estimating tests", besides the similarity transformation, you can use one more trick, we will consider the values of the sequence $y_{n}$ at the points $y_{0}, y_{1}, \ldots, y_{k-1}$ assuming that $y_{0}=0[14,15]$. It is easy to achieve this condition at any approximation step using a linear transformation:

$$
y_{0}=y_{j}-y_{j}, \quad y_{1}=y_{j+1}-y_{j}, \quad y_{2}=y_{j+2}-y_{j}, \ldots, \quad y_{k-1}=y_{j+k-1}-y_{j} .
$$

We will calculate coefficients of the linear, parabolic, logarithmic, and exponential approximation of the numerical sequence $y_{n}$ over the natural nodes $y_{0}, y_{1}, \ldots, y_{k-1}$.

First, using the method of least squares, we calculate the coefficients $a, b$ of the linear function $f(x)=a x+b$ approximating the natural nodes $y_{0}, y_{1}, \ldots, y_{k-1}$. For this, we find the local minimum of the function of two variables

$$
f_{l}(a, b)=\sum_{i=0}^{k-1}\left(a \cdot i+b-y_{i}\right)^{2} .
$$

Calculate the partial derivatives of the function $f_{l}(a, b)$

$$
\begin{gathered}
\frac{\partial f_{l}}{\partial a}=2 a \sum_{i=0}^{k-1} i^{2}+2 b \sum_{i=0}^{k-1} i-2 \sum_{i=0}^{k-1} i \cdot y_{i}, \\
\frac{\partial f_{l}}{\partial b}=2 a \sum_{i=0}^{k-1} i+2 b \sum_{i=0}^{k-1} 1-2 \sum_{i=0}^{k-1} y_{i} .
\end{gathered}
$$

By equating them to zero, we obtain a system of linear equations for the unknown $a$ and $b$ :

$$
\left\{\begin{array}{l}
\frac{k(k-1)(2 k-1)}{6} \cdot a+\frac{k(k-1)}{2} \cdot b=\sum_{i=1}^{k-1} i \cdot y_{i}, \\
\frac{k(k-1)}{2} \cdot a+k \cdot b=\sum_{i=1}^{k-1} y_{i}
\end{array}\right.
$$

which implies

$$
a=\frac{6}{k\left(k^{2}-1\right)} \sum_{i=1}^{k-1}(2 i+1-k) y_{i}, \quad b=\frac{2}{k(k+1)} \sum_{i=1}^{k-1}(2 k-1-3 i) y_{i} .
$$

Then we calculate the coefficients $c, d$ of the incomplete quadratic function $c x^{2}+d$ as the local minimum for

$$
f_{q}(c, d)=\sum_{i=0}^{k-1}\left(c \cdot i^{2}+d-y_{i}\right)^{2} .
$$

Differentiating $f_{q}(c, d)$ we find

$$
\frac{\partial f_{q}}{\partial c}=2 c \sum_{i=0}^{k-1} i^{4}+2 d \sum_{i=0}^{k-1} i^{2}-2 \sum_{i=0}^{k-1} i^{2} \cdot y_{i}
$$




$$
\begin{gathered}
\frac{\partial f_{q}}{\partial d}=2 c \sum_{i=0}^{k-1} i^{2}+2 d \sum_{i=0}^{k-1} 1-2 \sum_{i=0}^{k-1} y_{i}, \\
\left\{\begin{array}{l}
\frac{k(k-1)(2 k-1)\left(3 k^{2}-3 k-1\right)}{30} \cdot c+\frac{k(k-1)(2 k-1)}{6} \cdot d=\sum_{i=1}^{k-1} i^{2} \cdot y_{i}, \\
\frac{k(k-1)(2 k-1)}{6} \cdot c+k \cdot d=\sum_{i=1}^{k-1} y_{i} .
\end{array}\right.
\end{gathered}
$$

We find that

$$
\begin{gathered}
c=\frac{30}{k(k-1)(2 k-1)\left(8 k^{2}-3 k-11\right)} \sum_{i=1}^{k-1}\left(6 i^{2}-(k-1)(2 k-1)\right) y_{i}, \\
d=\frac{6}{k\left(8 k^{2}-3 k-11\right)} \sum_{i=1}^{k-1}\left(3 k(k-1)-1-5 i^{2}\right) y_{i} .
\end{gathered}
$$

Using the method of least squares, we calculate the coefficients of the approximating function $g \ln (x+1)+h$. We find the local minimum of the function

$$
f_{n}(g, h)=\sum_{i=0}^{k-1}\left(g \ln (x+1)+h-y_{i}\right)^{2} .
$$

We calculate the partial derivatives of the function $f_{n}(g, h)$

$$
\begin{gathered}
\frac{\partial f_{n}}{\partial g}=2 \sum_{i=0}^{k-1} \ln (i+1)\left(g \ln (i+1)+h-y_{i}\right), \\
\frac{\partial f_{n}}{\partial h}=2 \sum_{i=0}^{k-1}\left(g \ln (i+1)+h-y_{i}\right),
\end{gathered}
$$

and equate them to zero, we find the system of equations

$$
\left\{\begin{array}{l}
g \sum_{i=0}^{k-1} \ln ^{2}(i+1)+h \sum_{i=0}^{k-1} \ln (i+1)=\sum_{i=1}^{k-1} \ln (i+1) y_{i}, \\
g \sum_{i=0}^{k-1} \ln (i+1)+k h=\sum_{i=1}^{k-1} y_{i} .
\end{array}\right.
$$

We find that

$$
\begin{gathered}
g=\frac{k \cdot \sum_{i=1}^{k-1} \ln (i+1) y_{i}-\sum_{i=0}^{k-1} \ln (i+1) \cdot \sum_{i=1}^{k-1} y_{i}}{k \cdot \sum_{i=0}^{k-1} \ln ^{2}(i+1)-\left(\sum_{i=0}^{k-1} \ln (i+1)\right)^{2}} \\
h=\frac{\sum_{i=1}^{k-1} y_{i} \cdot \sum_{i=0}^{k-1} \ln ^{2}(i+1)-\sum_{i=0}^{k-1} \ln (i+1) \cdot \sum_{i=1}^{k-1} \ln (i+1) y_{i}}{k \cdot \sum_{i=0}^{k-1} \ln ^{2}(i+1)-\left(\sum_{i=0}^{k-1} \ln (i+1)\right)^{2}} .
\end{gathered}
$$


Similarly, we calculate the coefficients $p$ and $q$ for the function $p e^{x}+q$, through finding the local minimum of the function

$$
f_{e}(p, q)=\sum_{i=0}^{k-1}\left(p e^{i}+q-y_{i}\right)^{2} .
$$

We calculate the partial derivatives of $f_{e}(p, q)$, equate them to zero, and solve the system of linear equations

$$
\begin{gathered}
\frac{\partial f_{e}}{\partial p}=2 \sum_{i=0}^{k-1} e^{i}\left(p e^{i}+q-y_{i}\right), \\
\frac{\partial f_{e}}{\partial q}=2 \sum_{i=0}^{k-1}\left(p e^{i}+q-y_{i}\right), \\
p \cdot \sum_{i=0}^{k-1} e^{2 i}+q \cdot \sum_{i=0}^{k-1} e^{i}=\sum_{i=1}^{k-1} e^{i} y_{i}, \\
p \cdot \sum_{i=0}^{k-1} e^{i}+k q=\sum_{i=1}^{k-1} y_{i} .
\end{gathered}
$$

We find that

$$
\begin{gathered}
p=\frac{k \cdot \sum_{i=1}^{k-1} e^{i} y_{i}-\sum_{i=0}^{k-1} e^{i} \cdot \sum_{i=1}^{k-1} y_{i}}{k \cdot \sum_{i=0}^{k-1} e^{2 i}-\left(\sum_{i=0}^{k-1} e^{i}\right)^{2}} \\
q=\frac{\sum_{i=1}^{k-1} y_{i} \cdot \sum_{i=0}^{k-1} e^{2 i}-\sum_{i=0}^{k-1} e^{i} \cdot \sum_{i=1}^{k-1} e^{i} y_{i}}{k \cdot \sum_{i=0}^{k-1} e^{2 i}-\left(\sum_{i=0}^{k-1} e^{i}\right)^{2}} .
\end{gathered}
$$

Now it is possible to construct three "approximation-estimating tests", which are designed to determine the moment when the increase in the monotonous sequence $y_{n}$, the numerical parameters of the solid, changes from linear to parabolic, logarithmic or exponential.

"Parabolic approximation-estimating test" has the form

$$
\delta_{l q}^{2}(k)=\delta_{l}^{2}(k)-\delta_{q}^{2}(k) .
$$

If for the natural nodes $y_{0}, y_{1}, \ldots, y_{k-1}$ the inequality $\delta_{l q}^{2}(k) \leqslant 0$ is satisfied, and for the nodes $y_{1}, y_{2}, \ldots, y_{k}$, shifted right by one step of discreteness, the inequality sign changes to the inverse $\delta_{l q}^{2}(k)>0$, then we can say that near the point $y_{k}$ the character of increasing of the sequence $y_{n}$ has changed from linear to parabolic.

Similarly, we define:

"logarithmic approximation-estimating test":

$$
\delta_{l n}^{2}(k)=\delta_{l}^{2}(k)-\delta_{n}^{2}(k),
$$

"exponential approximation-estimating test":

$$
\delta_{l e}^{2}(k)=\delta_{l}^{2}(k)-\delta_{e}^{2}(k) .
$$


4. Markov's moments for the boundaries of elastic deformation. Consider a physical or numerical experiment as a random process.

Let $T=\overline{1, m-1}$, a bounded subset of the natural series containing the first $m-1$ natural numbers (we note right away that $m$ can be arbitrarily large). Then the indexed family $\xi=\left\{\xi_{t}, t \in T\right\}$ of random variables $\xi_{t}=\xi_{t}(\omega)$ given for $\forall t \in T$ on the same probability space $(\Omega, \mathcal{F}, \mathrm{P})$ is called a discrete random process $[16,17]$.

Each random variable $\xi_{t}$ generates an $\sigma$-algebra, which will be denoted as $\mathcal{F}_{\xi_{t}}$. Then the $\sigma$-algebra generated by the random process $\xi=\left\{\xi_{t}, t \in T\right\}$ is the smallest $\sigma$-algebra containing all $\mathcal{F}_{\xi_{t}}$ that is

$$
\sigma(\xi)=\sigma\left(\bigcup_{t=1}^{m-1} \mathcal{F}_{\xi_{t}}\right) .
$$

A discrete random process $\xi=\left\{\xi_{t}, t \in T\right\}$ can be considered as a function of two variables $\xi=\xi(t, \omega)$, where $t$ is a natural the argument $\omega$ is a random event. If we fix $t$, then, as mentioned above, we get a random variable $\xi_{t}$, but if we fix a random event $\omega_{0}$, we get a function of the natural argument $t$, which is called the trajectory of the random process $\xi=\left\{\xi_{t}, t \in T\right\}$ and is a random sequence $\xi_{t}\left(\omega_{0}\right)$.

We will consider only those random processes whose trajectories monotonically increase. An arbitrary random $\omega \in \Omega$ event is to extract a sample $X$ from the $n$-dimensional Euclidean space $\mathbb{E}^{n}$. Theoretically, any point $\bar{x} \in \mathbb{E}^{n}$ can belong to the sampling $X$, therefore, the $\sigma$-algebra from the probability space $(\Omega, \mathcal{F}, \mathrm{P})$ contains $\mathbb{E}^{n}$, any finite set $X$ from the space $\mathbb{E}^{n}$, all possible countable unions of such sets, and additions to by him. Denote this system of sets as $\mathcal{S}\left(\mathbb{E}^{n}\right)$ and call it "selective $\sigma$-algebra" $\mathcal{F}=\mathcal{S}\left(\mathbb{E}^{n}\right)$. The same reasoning is valid for any $\sigma$-algebra $\mathcal{F}_{\xi_{t}}$, therefore,

$$
\sigma(\xi)=\mathcal{S}\left(\mathbb{E}^{n}\right)
$$

Note that this $\sigma$-algebra is "poorer" than the Borel $\sigma$-algebra $-\mathcal{S}\left(\mathbb{E}^{n}\right) \subset \mathcal{B}\left(\mathbb{E}^{n}\right)$. Indeed, a countable union of at most countable sets is countable, therefore, $\mathcal{S}\left(\mathbb{E}^{n}\right)$ does not contain intervals.

We will consider the problem of testing statistical hypotheses $H_{0}$ and $H_{1}$ [18]. There are two hypotheses $H_{0}$ : "the random sequence $\xi_{t}\left(\omega_{0}\right)$ increases is linear", and $H_{1}$ : "the random sequence $\xi_{t}\left(\omega_{0}\right)$, increases is nonlinearly". $H_{0}$ is called the null hypothesis, $H_{1}$ is called the alternative hypothesis.

It is necessary to construct a criterion as a strict mathematical rule, to test the statistical hypothesis. That rule accepts or rejects the hypothesis. Any statistical criterion is based on a random sample $X$. Two cases are possible. The first one, a sample $X$, is extracted from $\mathbb{E}^{n}$ simultaneously and has a fixed size. The second one, when a sample $X$ form at the period. Then its size is a random variable. In this case, the sequential analysis and the construction of a stopping time are used [19-21].

Let $(\Omega, \mathcal{F}, \mathrm{P})$ be a probability space, then the indexed family of $\sigma$-algebras $\mathfrak{F}=\left\{\mathcal{F}_{t}, t \in T\right\}$ is called a filtration if for $\forall i, j \in T \mid i<j: \mathcal{F}_{i} \subset \mathcal{F}_{j} \subset \mathcal{F}$. Moreover, if for $\forall t \in T$ right $\mathcal{F}_{t}=\sigma\left(\xi_{i}, i<t\right)$, then the filtration is called natural. The random process $\xi=\left\{\xi_{t}, t \in T\right\}$ is said to be adapted to the filtration $\mathfrak{F}$ if for $\forall t \in T: \sigma\left(\xi_{t}\right)=\mathcal{F}_{\xi_{t}} \subset \mathcal{F}_{t}$.

Any stochastic process $\xi=\left\{\xi_{t}, t \in T\right\}$ is an adapted process with respect to its natural filtration. The mapping $\tau: \Omega \longrightarrow T$ is called Markov moment [22] or stopping time (with respect to the filtration $\mathfrak{F}$ ) if for $\forall t \in T$ the preimage of the set $\{\tau \leqslant t\} \in \mathcal{F}_{t}$. In other words, let $\tau$ be the moment of occurrence of some event in the random process $\xi=\left\{\xi_{t}, t \in T\right\}$. If for $\forall t_{0} \in T$, you can definitely say if the event $\tau$ has come or not, 
provided that the values of $\xi_{t}$ are known only in the past (to the left of $t_{0}$ ), then $\tau$ is a Markov moment with respect to natural filtration of $\mathfrak{F}$, random process $\xi=\left\{\xi_{t}, t \in T\right\}[21$, $23]$. In this case for a random sequence $\xi_{t}\left(\omega_{0}\right)$ the natural filtration an adapted with process is it the selective $\sigma$-algebra $\mathcal{S}\left(\mathbb{E}^{n}\right)$.

In the case of the sequential statistical analysis, one can define the Markov moment (or stopping time) of the experiment as the values of $t$ in which the change occurred like an increase in the random sequence $\xi_{t}\left(\omega_{0}\right)$ from linear type to nonlinear type. As such, we reject the null hypothesis $H_{0}$ and accept the alternative hypothesis $H_{1}$. We write out the values of $\tau$ explicitly.

The transition from linear increase to parabolic growth - the Markov moment:

$$
\tau=\min \left\{t \mid \delta_{l q}^{2}(k)>0\right\} .
$$

The transition from linear increase to logarithmic growth - the Markov moment:

$$
\tau=\min \left\{t \mid \delta_{l n}^{2}(k)>0\right\} .
$$

The transition from a linear increase to an exponential growth - the Markov moment:

$$
\tau=\min \left\{t \mid \delta_{l e}^{2}(k)>0\right\} .
$$

5. Numerical simulation example. Let us consider the experiment results on uniform bending by a concentrated force $P$ of a cantilever beam of model material with a single step of discreteness. It is required to determine the point in time when elastic deformation turns into plastic deformation. We use the hypothesis that the beginning of the plasticity development coincides with the point in time when the strains begin to increase rapidly, and their dependence on the load ceases to be linear. We will write a "parabolic approximation-estimating test" previously constructed for four natural nodes [14, 15]:

$$
\delta_{l q}^{2}(4)=\frac{1}{245}\left(19 y_{1}^{2}-11 y_{2}^{2}+41 y_{3}^{2}+12 y_{1} y_{2}-64 y_{1} y_{3}-46 y_{2} y_{3}\right)
$$

The deformation values and values of the criterion $\delta^{2}(4)$ are given in the Table. Values $y_{p}$ present in a dimensionless and normalized form. The stress is also normalized and increases in the unit step. The symbol $\delta_{4}^{2}$ denotes the criterion value under natural nodes: $y_{1}, y_{2}, y_{3}, y_{4}$, the symbol $\delta_{5}^{2}$ under nodes: $y_{2}, y_{3}, y_{4}, y_{5}$, etc.

The first members of $y_{n}$ is increase almost linear, since $\delta_{l q}^{2}=\delta_{l}^{2}-\delta_{q}^{2}<0$. Sign becomes positive of the quadratic form $\delta_{l q}^{2}$ for nodes: $y_{26}=15.90, y_{27}=16.47, y_{28}=17.26, y_{29}=$ 18.62. It means that the character of increasing $y_{n}$ has changed and has become parabolic. The graph of the numerical sequence $y_{n}$, shown in Figure, confirms this.

6. Conclusion. "Approximation-estimation tests" allow, with some probability, to determine the Markov moment in a physical or numerical experiment when the limit of applicability of Hooke's law is reached. A significant problem is related to the sensitivity of "approximation-estimating tests" toward the length of the current interval of approximation and step of discreteness. This problem is that by a tiny step of discreteness, the change in any monotonic sequence will be linear, and to detect the occurrence of nonlinearity, it is necessary to increase the length of the current interval of approximation. Indeed, an increment of any smooth function is an infinitesimal quantity. It has been known that, in a small neighborhood, this increment is accurately approximated by the differential. However, as the argument increments increase, the differential approximation ceases to be satisfactory. Therefore, there is no general answer to the problem posed. It 
Table. Values of the strain $y_{p}$ and values the criterion $\delta^{2}(4)$

\begin{tabular}{|c|c|c|c|c|}
\hline Step number & Strain id & Strain value & Criterion id & Criterion value \\
\hline 1 & $y_{1}$ & 1.00 & - & - \\
2 & $y_{2}$ & 1.51 & - & - \\
3 & $y_{3}$ & 2.03 & - & - \\
4 & $y_{4}$ & 2.54 & $\delta_{4}^{2}$ & -0.11 \\
5 & $y_{5}$ & 3.05 & $\delta_{5}^{2}$ & -0.11 \\
6 & $y_{6}$ & 3.57 & $\delta_{6}^{2}$ & -0.11 \\
7 & $y_{7}$ & 4.08 & $\delta_{7}^{2}$ & -0.11 \\
8 & $y_{8}$ & 4.59 & $\delta_{8}^{2}$ & -0.11 \\
9 & $y_{9}$ & 5.10 & $\delta_{9}^{2}$ & -0.11 \\
10 & $y_{10}$ & 5.74 & $\delta_{10}^{2}$ & -0.08 \\
11 & $y_{11}$ & 6.25 & $\delta_{11}^{2}$ & -0.13 \\
12 & $y_{12}$ & 6.76 & $\delta_{12}^{2}$ & -0.16 \\
13 & $y_{13}$ & 7.27 & $\delta_{13}^{2}$ & -0.11 \\
14 & $y_{14}$ & 8.08 & $\delta_{14}^{2}$ & -0.04 \\
15 & $y_{15}$ & 8.60 & $\delta_{15}^{2}$ & -0.16 \\
16 & $y_{16}$ & 9.11 & $\delta_{16}^{2}$ & -0.26 \\
17 & $y_{17}$ & 9.79 & $\delta_{17}^{2}$ & -0.07 \\
18 & $y_{18}$ & 10.30 & $\delta_{18}^{2}$ & -0.14 \\
19 & $y_{19}$ & 11.03 & $\delta_{19}^{2}$ & -0.14 \\
20 & $y_{20}$ & 11.61 & $\delta_{20}^{2}$ & -0.13 \\
21 & $y_{21}$ & 12.26 & $\delta_{21}^{2}$ & -0.20 \\
22 & $y_{22}$ & 13.29 & $\delta_{22}^{2}$ & -0.02 \\
23 & $y_{23}$ & 13.86 & $\delta_{23}^{2}$ & -0.28 \\
24 & $y_{24}$ & 14.63 & $\delta_{24}^{2}$ & -0.36 \\
25 & $y_{25}$ & 15.00 & $\delta_{25}^{2}$ & -0.21 \\
26 & $y_{26}$ & 15.90 & $\delta_{26}^{2}$ & -0.12 \\
27 & $y_{27}$ & 16.47 & $\delta_{27}^{2}$ & -0.09 \\
28 & $y_{28}$ & 17.26 & $\delta_{28}^{2}$ & -0.27 \\
29 & $y_{29}$ & 18.62 & $\delta_{29}^{2}$ & 0.14 \\
30 & $y_{30}$ & 20.52 & - & - \\
31 & $y_{31}$ & 23.46 & - & - \\
32 & $y_{32}$ & 28.17 & - & - \\
\hline & & & & \\
\hline
\end{tabular}

\section{Strain}

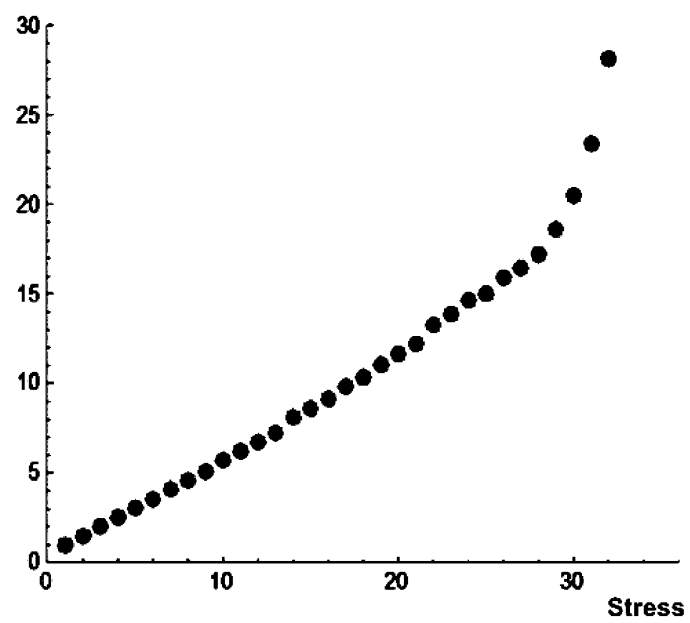

Figure. Strain-stress curve for uniform bending by a concentrated force $P$ of a cantilever beam 
should bear in mind that the increase in the number of natural nodes of approximation inevitably entails a geometric increase in the computational complexity of constructing a quadratic form for these tests.

The following thought gives some relief to the mind. If the discreteness step is so small that the approximation parameters of the stress-strain state of a solid still change linearly in the current interval, then the corresponding mathematical models can be built within the paradigm of generalized Hooke's law. On the other hand, proposed "approximationestimating tests" allow us to determine the boundaries beyond which Hooke's law's use becomes incorrect. By the way, this law has usually used for small deformations. Therefore, admitting freedom of speech, it can be said that the "approximation-estimating tests" allow not only to determine the boundaries of the application of Hooke's law but also to formally approach the determination of the quantitative value of small deformation, for each fixed structural material.

\section{References}

1. Timoshenko S. Strength of materials. 3rd ed. Malabar, Florida, Krieger Publ. Co., 1983, 1010 p.

2. Beer F., Russell J. Jr. E., DeWolf J., Mazurek D. Mechanics of materials. 7th ed. New York, McGraw Hill Education Publ., 2014, 896 p.

3. Friedman Ya. B. Mekhanicheskiye svoystva materialov. Ch. 1. Deformatsiya $i$ razrusheniye. [Mechanical properties of metals. Pt 1. The deformation and fracture]. Moscow, Mashinostroyeniye Publ., 1974, 472 p. (In Russian)

4. Schneider S., Schneider S. G., da Silva H. M., De Moura Neto C. Study of the non-linear stressstrain behavior in Ti-Nb-Zr alloys. Materials Research [Online], 2005, vol. 8, pp. 435-438. https://doi.org/10.1590/S1516-14392005000400013

5. Schneider S. G., Nunes C. A., Rogero S. O., Higa O. Z., Bressianil J. C. Mechanical properties and cytotoxic evaluation of the Ti-3Nb-13Zr alloy. Biomechanica, 2000, vol. 8(1), pp. 84-87.

6. Pavilainen G. V., Yushin R. Yu. Analiz ucheta uprugoy transversal'noy izotropii i plasticheskoy anizotropii pri izgibe kruglykh plastin [Analysis of taking into account elastic transversal isotropy and plastic anisotropy when bending round plates]. Vestnik of Saint Petersburg University. Series 1. Mathematics. Mechanics. Astronomy, 2011, iss. 1, pp. 122-131. (In Russian)

7. Pavilainen G. V., Yushin R. Yu. An approximate solution of elastic-plastic problem of circular strength different (SD) plates. CNSA 201\%. Constructive Nonsmooth Analysis and Related Topics (dedicated to the memory of V. F. Demyanov) (CNSA). IEEE, 2017, pp. 7973999.

https://doi.org/10.1109/CNSA.2017.7973999

8. Atlas of stress-strain curves. 2nd ed. United States, ASM International Press, 2002, 816 p.

9. Sluzalec A. Stress-strain curve. Introduction to nonlinear thermomechanics. London, Springer Press, 1992, pp. 45-47.

10. Kariya T., Kurata H. Generalized least squares. England, Chichester, West Sussex, John Wiley \& Sons, Ltd. Publ., 2004, 307 p. 344 p.

11. Luenberger D. G. Optimization by vector space methods. London, Wiley-Interscience Press, 1969,

12. Lang S. Algebra. New York, Springer-Verlag Press, 2002, xv +914 p.

13. Shimura G. Arithmetic of quadratic forms. New York, Springer-Verlag Press, 2010, xii $+238 \mathrm{p}$.

14. Orekhov A. V. Criterion for estimation of stress-deformed state of SD-materials. AIP Conference Proceedings, AIP Publ., 2018, vol. 1959, pp. 070028. https://doi.org/10.1063/1.5034703

15. Orekhov A. V. Approksimatsionno-otsenochnyye kriterii napryazhenno-deformiruyemogo sostoyaniya tverdogo tela [Approximation-evaluation tests for a stress-strain state of defor- mable solids]. Vestnik of Saint Petersburg University. Applied Mathematics. Computer Science. Control Processes, 2018, vol. 14, iss. 3, pp. 230-242. https://doi.org/10.21638/11702/spbu10.2018.304 (In Russian)

16. Krishnan V. Probability and random processes. Hoboken, New Jersey, John Wiley \& Sons, Inc. Publ., 2006, 723 p.

17. Ibe O. Fundamentals of applied probability and random processes. San Diego, Academic Press, 2014, $456 \mathrm{p}$.

18. Lehmann E. L., Romano J. P. Testing Statistical Hypotheses. New York, Springer-Verlag Press, 2005 , xiv +786 p.

19. Wald A. Sequential Analysis. New York, John Wiley \& Sons, Inc. Publ., 1947, 212 p. 
20. Chow Y. S. Great expectations: the theory of optimal stopping. Boston, Houghton Mifflin Press, 1971, $139 \mathrm{p}$.

21. Sirjaev A. N. Statistical sequential analysis: Optimal stopping rules. New York, American Mathematical Society Press, 1973, 174 p.

22. Chung K. L. Lectures from Markov processes to Brownian (Grundlehren Der Mathematischen Wissenschaften). New York, Springer-Verlag Press, 1982, vii+242 p.

23. Shiryaev A. N. Optimal stopping rules. Berlin, Heidelberg, Springer-Verlag Press, 2008, xii+220 p. https://doi.org/10.1007/978-3-540-74011-7

Received: October 18, 2020.

Accepted: October 23, 2020 .

Authors' information:

Andrey V. Orekhov - Senior Lecturer; a_v_orehov@mail.ru

\section{Статистические критерии для пределов применения закона Гука}

\section{A. B. Орехов}

Санкт-Петербургский государственный университет, Российская Федерация, 199034, Санкт-Петербург, Университетская наб., 7-9

Для цитирования: Orekhov A. V. Statistical criteria for the limits of application of Hooke's law // Вестник Санкт-Петербургского университета. Прикладная математика. Информатика. Процессы управления. 2020. Т. 16. Вып. 4. С. 391-401.

https://doi.org/10.21638/11701/spbu10.2020.404

При современных способах исследования напряженно-деформированного состояния твердых тел и определения точки перехода от упругой к пластической деформации (точки предела упругости) используются графические методы, основанные на визуальной оценке кривых напряжения-деформации (диаграмм деформирования). Однако этот подход не является формальным и предназначен только для ситуаций, когда напряжение - это функция от деформации в одномерном случае. Случаи, когда деформация есть функция от нагрузки, также имеют большое практическое значение. Цель статьи построение формальных правил для определения границ применимости закона Гука. Предлагаемые аналитические методы основаны на последовательном статистическом анализе. Выведены квадратичные формы для вычисления точки, в которой тип возрастающей монотонной числовой последовательности изменяется от линейного к нелинейному. С помощью таких квадратичных форм строятся статистические критерии для оценки границ применимости закона Гука, которые определяются как марковские моменты. Новизна результатов состоит в том, что предел упругости можно вычислить без визуализации экспериментальных данных. Приводится численный пример применения параболического аппроксимационно-оценочного критерия. По результатам этого эксперимента можно сделать вывод, что аналитическое определение границ применимости закона Гука совпадает с визуальной оценкой и аппроксимационно-оценочные критерии позволяют формально вычислять пределы применимости закона Гука.

Ключевые слова: закон Гука, напряжение, деформация, аппроксимационно-оценочный критерий, метод наименьших квадратов, марковский момент.

Контактная информация:

Орехов Андрей Владимирович - ст. преп.; a_v_orehov@mail.ru 